\title{
Individuation of object parts in aging
}

\section{Chiara F. Tagliabue $^{1}$ (D) $\cdot$ Luigi Lombardi ${ }^{2} \cdot$ Veronica Mazza $^{1}$}

Published online: 4 March 2020

(C) The Psychonomic Society, Inc. 2020

\begin{abstract}
Research on enumeration with isolated objects has indicated that young and older adults can report up to three elements with similar efficiency (subitizing effect). Recent studies on subitizing in young adults have shown that individuation occurs over parts of an object as efficiently as over physically disconnected objects, suggesting that spatial separation is a sufficient requirement for efficient individuation. Do young and older adults share this sufficient requirement? In two experiments, we tested for the presence of subitizing in an enumeration task with a varying number of distinct objects and object parts. In Experiment 1, results indicated the presence of a bilinear function (with an inflection point between 3 and 4 elements, a proxy for subitizing) in the response speed of young and older adults, and in both stimulus conditions. In addition, the enumeration slope in older participants was steeper for object parts than for objects in the subitizing range, possibly due to perceptual degradation (e.g., in contour detection). The pattern found generalizes to other stimuli (Experiment 2), thus highlighting the robustness of the present findings. Overall, the results indicate that while some perceptual factors (such as contour detection or curvature polarity) may hamper subitizing speed of older individuals relative to young adults, the subitizing span remains at approximately three to four elements for multiple objects and object parts in both young and older adults. Thus, individuation of multiple objects and object parts is a mechanism relatively resistant to aging.
\end{abstract}

Keywords Individuation $\cdot$ Aging $\cdot$ Subitizing $\cdot$ Object parts $\cdot$ Enumeration

\section{Introduction}

Cognitive deterioration is a sign of physiological aging (Salthouse, 2004). For instance, in memory tasks older participants typically retain less information compared to young individuals (Park et al., 2002), and are overall slower in task execution (Rabbitt, 2016; Salthouse, 1996, 2004). An intriguing exception to this trend is represented by enumeration tasks, in which the performance of older and young individuals is equivalent. When the items to be enumerated are presented without distracting information, older participants can enumerate up to three to four elements as efficiently as young

Electronic supplementary material The online version of this article (https://doi.org/10.3758/s13414-020-01996-2) contains supplementary material, which is available to authorized users.

Chiara F. Tagliabue

chiara.tagliabue@unitn.it

1 Center for Mind/Brain Sciences (CIMeC), University of Trento, Corso Bettini 31, 38068 Rovereto, TN, Italy

2 Department of Psychology and Cognitive Science, University of Trento, Trento, Italy individuals, a phenomenon known as subitizing (Basak \& Verhaegen, 2003; Pagano et al., 2016; Trick et al., 1996; Watson et al., 2002).

Subitizing is the fast and accurate enumeration of a small set of elements as compared to slower and/or less accurate enumeration ("counting") for larger sets of elements (Mandler \& Shebo, 1982). The most pervasive explanation of subitizing points to the existence of an objectindividuation mechanism that allows efficient tracking of a limited set of items (Pylyshyn, 2001; Trick \& Pylyshyn, 1993, 1994). Individuation is the ability to process each element of a set as being separated from other elements. Although its processing structure remains to be fully determined, individuation can result in a set of more robust representations of the objects (e.g., Kahneman, Treisman, \& Gibbs, 1992), making them available for further processing. As such, it seems to be the logical prerequisite for enumeration, and for the emergence of subitizing. Early proposals argued that object individuation operates separately from attention (Trick \& Pylyshyn, 1993), but recent research has suggested that simultaneous indexing of relevant items in a scene is tightly related to attention (Cavanagh, 2011; Mazza \& Caramazza, 2011; Vetter et al., 2008). For example, subitizing disappears in dual tasks when the primary task becomes more demanding (Vetter 
et al., 2008). Thus, variation in the attentional load imposed by the task largely modulates the occurrence of the subitizing effect. The fact that subitizing is preserved in aging in easy perceptual contexts (such as in the case of targets presented in isolation) suggests that for tasks requiring relatively minimal levels of attention involvement, older individuals are able to individuate (a limited set of) multiple items as efficiently as younger adults (Trick et al., 1996).

In the context of subitizing, individuation has been suggested to function on "objects" intended as physically disconnected entities. For instance, Trick and Pylyshyn (1993) showed that, when connected items (such as elements presented in a grid of lines) are used, no subitizing emerged. Similarly, subitizing does not seem to occur over non-spatial features. For example, when adults have to enumerate the number of colors presented in a display, and these are uncorrelated with the number of objects, there is no subitizing effect (Watson et al., 2005a). Thus, early studies seem to indicate that efficient individuation does not occur when non-spatial features (such as color) are used. However, research on attention cueing (e.g., Vecera et al., 2000) has indicated that the typical validity effect (wherein cued elements are processed more efficiently than uncued elements) is also present for parts of an object, similar to what occurs with totally disconnected items. On the basis of these results, it was proposed that attention can select either objects or their parts. Accordingly, other studies have shown that the typical advantage related to "objects" can be found with and without connectivity, leading to the conclusion that there is no qualitatively special role for disconnected objects in attention selection (Marino \& Scholl, 2005).

In line with these findings, recent evidence on enumeration tasks contradicts the view that the units of individuation in subitizing are only spatially disconnected items, and suggests that spatial separability is sufficient requirement for efficient individuation. For instance, Porter et al. (2016) found that multiple parts bound to a single object can be enumerated as efficiently as multiple distinct objects. In addition, a neural signature of target individuation varies as a function of objectpart numerosity in the same way as in the case of distinct objects, suggesting that the same neural mechanism triggers individuation of multiple objects and multiple parts of a single object (Poncet et al., 2016; Wurm et al., 2019).

Does aging affect the level of sufficient requirement for individuation in enumeration? In other words, do older adults retain the ability to individuate object parts efficiently (as in the subitizing effect), or is this ability impaired? Individuation of object parts may be critically affected by aging, given that some (although not all) perceptual processes deteriorate with aging. Visual acuity, contrast sensitivity and contour extraction are among the perceptual dimensions that are modified by aging (Monge \& Madden, 2016; Sekuler \& Sekuler, 2000). These age-related changes could lead to impairments in isolating connected parts of an object, and thus to the ability to enumerate them efficiently, compared to disconnected objects. Contour detection could be particularly important in order to integrate information coming from multiple parts of an object into a coherent unit (the "object"; see Roudaia et al., 2008,2011 ). For instance, older adults require longer stimulus duration to discriminate between or detect global contours (Roudaia et al., 2011). As older adults are typically less efficient in contour detection (Andersen \& Ni, 2008; Del Viva \& Agostini, 2007, Roudaia et al., 2008, 2011), they may require a large amount of resources to distinguish the various parts of the object as being such (see Fig. 1A). Thus, the ability of elderly individuals to enumerate efficiently the multiple parts of an object relative to distinct objects may be impaired.

To evaluate whether and how aging affects efficient individuation of object parts, we conducted two experiments where participants enumerated a varying number of either objects or object parts, as in Porter et al. (2016). In Experiment 1, a group of "young" and "older" participants reported the number of either distinct objects or object parts (protrusions of a circle). The aim of Experiment 2 was to generalize the findings obtained in the older sample for object parts to other stimuli, just as it has already been shown for young adults (see Porter et al., 2016). For this reason, here protrusions and concavities were used as targets.

\section{Methods}

\section{Participants}

Eighteen young (mean age 22.4 years, range 19-27 years, 11 female) and 20 older (mean age 67.6 years, range $61-76$ years, 9 female) adults volunteered for Experiment 1. Eighteen young (mean age 23.3 years, range 19-27, 14 female) and 20 older volunteers (mean age 68.1 years, range $63-77$ years, 10 female) were tested in Experiment 2. The sample size was determined based on previous studies (Poncet et al., 2016; Porter et al., 2016; Trick et al., 1996; Watson et al., 2005b). No participant reported a history of neurological or psychiatric disorders. All participants had normal or corrected-to-normal vision and provided written informed consent. The procedures were approved by the University of Trento Ethics Committee. Both groups of older participants underwent a session of neuropsychological tests to assess their cognitive integrity, with a focus on visuospatial abilities (see Table 1).

Stimuli and procedure In Experiment 1, the stimuli and procedure were similar to the ones used in Porter et al. (2016, Experiment 6). Single-object configurations (protrusions) consisted of a black outline circle with protruding arcs presented over a gray background. Multiple-object configurations (shapes) consisted of outline arcs (Fig. 1A). The 
a

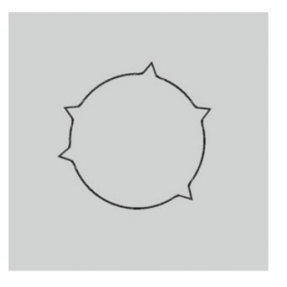

b

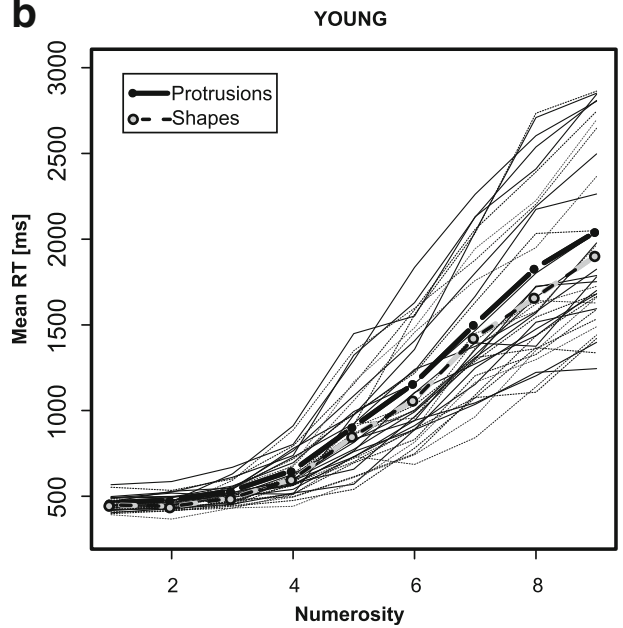

Fig. 1. (A) Example of stimuli for Experiment 1: protrusions (left) and shapes (right). Mean reaction times as a function of numerosity and stimulus condition for (B) young and (C) older participants. The thick solid

locations of the arcs in the single-object configurations were generated randomly for each participant, with the constraint that no feature overlapping occurred. The orientation of the arcs in the multiple-object configurations was selected randomly from a set of angles $\left(-20^{\circ},-10^{\circ}, 0^{\circ},+10^{\circ},+20^{\circ}\right)$ to avoid the illusory percept of a circle. Visual stimuli subtended a $6.7^{\circ}$ visual angle.
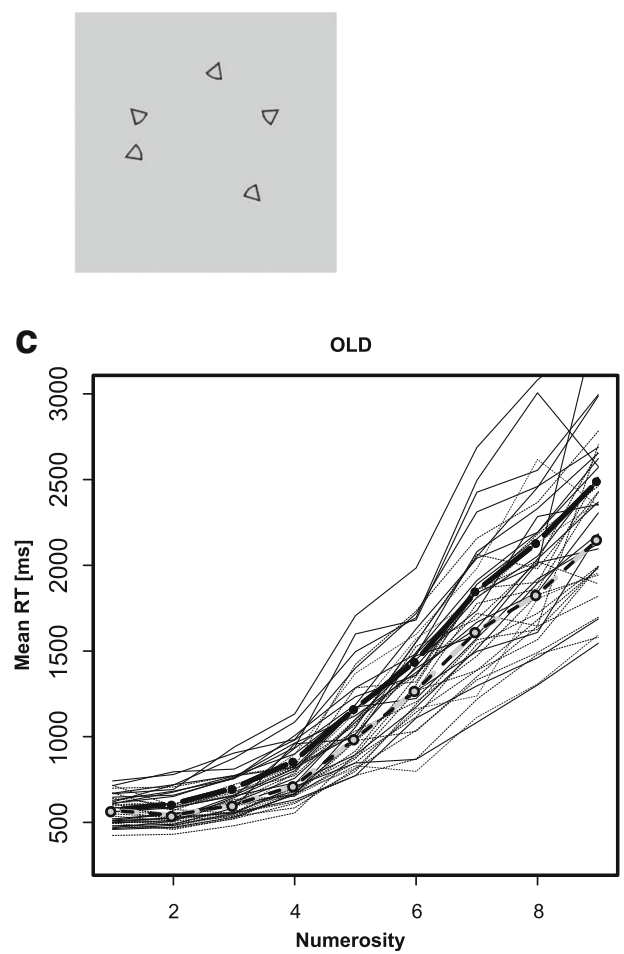

(protrusions) and segmented (shapes) lines represent the group average, while the thin solid and segmented lines represent each individual participant

In Experiment 2, the stimuli and procedure were also similar to the ones used in Porter et al. (2016, Experiment 3). Two types of single-object configurations were created. In the "concavity" condition, a varying number of gray semicircles (same color as background) overlapped with a central black circle, creating the perception of a black circle with missing parts; in the "protrusion" condition, a varying number of black

Table 1. Mean and median raw scores obtained at each neuropsychological test by the older groups

\begin{tabular}{|c|c|c|c|c|}
\hline \multirow[b]{2}{*}{ Neuropsychological tests } & \multicolumn{2}{|c|}{ Experiment 1} & \multicolumn{2}{|l|}{ Experiment 2} \\
\hline & Mean (SD) & Median & Mean (SD) & Median \\
\hline Mini Mental State Examination & $29.2(1.4)$ & 30 & $29.5(0.9)$ & 30 \\
\hline Raven's Coloured Progressive Matrices 47 & $32.5(2.8)$ & 33 & $33.8(2.4)$ & 34.5 \\
\hline Attentive Matrices (Visual Search) & $55.5(4.6)$ & 57 & $55.3(3.3)$ & 56 \\
\hline Trail Making Test A & $39.3(11.9)$ & 40 & $38.4(9.9)$ & 38 \\
\hline Trail Making Test B & $87.2(25.8)$ & 75 & $102.3(43.2)$ & 96.5 \\
\hline Phonemic fluency & $44.1(15.9)$ & 39 & $41.3(12.4)$ & 41 \\
\hline Digit Span Forward & $5.9(0.9)$ & 6 & $6.2(0.9)$ & 6 \\
\hline Digit Span Backward & $4.5(1.1)$ & 4 & $4.9(1.3)$ & 4.5 \\
\hline $\begin{array}{l}\text { Rey-Osterrieth complex figure } \\
\text { copy }\end{array}$ & $33.1(2.3)$ & 33 & $33.3(2.7)$ & 34 \\
\hline $\begin{array}{l}\text { Rey-Osterrieth complex figure } \\
\text { recall }\end{array}$ & $17(4.8)$ & 19 & $17.4(4.6)$ & 18.3 \\
\hline Geriatric Depression Scale & $5.4(3.3)$ & 5 & $4.9(3.9)$ & 4.5 \\
\hline
\end{tabular}


semicircles extended from the central black circle. The locations of the arcs in the single-object configuration were generated for each participant in such a way that no target feature overlapping was possible. Visual stimuli subtended a $6.7^{\circ}$ visual angle.

In both experiments, each trial consisted of a fixation display ( $1 \mathrm{~s}$ ) followed by the stimulus display (for a maximum of 4 s). Participants were instructed to report the number of targets (Experiment 1: either protrusions or shapes; Experiment 2: either protrusions or concavities) by pressing the space bar on the keyboard and by saying aloud their response (Atkinson et al., 1976; Watson \& Humphreys, 1999; Watson et al., 2002, 2005a, b; Maylor et al., 2005, 2008; Porter et al., 2016). The experimenter recorded the responses via the number keys on the keyboard. This procedure was followed given that it proved successful in our own studies (Porter et al., 2016), as well as in some previous studies on enumeration in the older population (see Maylor et al., 2005, 2008).

A blank screen $(1 \mathrm{~s})$ terminated the trial. Participants completed a practice session with two blocks (Experiment 1: protrusions vs. shapes; Experiment 2: concavities vs. protrusions) of ten trials each. In the experimental session, the two stimulus configurations (Experiment 1: protrusions vs. shapes; Experiment 2: concavities vs. protrusions) were presented consecutively in six blocks with an AAABBB design (20 trials for each numerosity, overall 180 trials per configuration). The order of presentation of the configurations was counterbalanced across participants. The order of the displays within a block was determined pseudorandomly, with the constraint that no two identical displays were presented in sequence.

Statistical analysis Given the relatively long stimulus duration (up to $4 \mathrm{~s}$ ), which typically leads to higher accuracy levels (here, above $96 \%$ on average), we considered response times (RTs) as the main variable of interest (see also Porter et al., 2016). Analyses on the proportion of correct responses of both experiments are reported in the Supplementary Material. Trials with incorrect responses and with RTs above \pm 2 standard deviations from the mean (e.g., Porter et al., 2016) were discarded from the analysis (on average, $4.7 \%$ of discarded trials in both experiments). For each participant in the two groups, individual mean RTs were fitted by performing both a linear and a bilinear regression model with numerosity, stimulus type (two levels: protrusions and shapes for Experiment 1, protrusions and concavities for Experiment 2), and the interaction term (numerosity $\times$ stimulus type) as independent variables. In particular, with respect to the bilinear regression analysis, all the model parameters (inflection-points, preinflection slopes and post-inflection slopes) were estimated on individual participant data using the $\mathrm{R}$ statistical package segmented (Muggeo, 2008). The overall performance of the models (linear and bilinear regressions) was evaluated separately for each group using the adjusted $\mathrm{R}^{2}$, Akaike information (AIC) and Bayesian information criterion (BIC).

Furthermore, we conducted separate analyses with linear mixed models (LMMs) on each estimated parameter (inflection-points, pre-inflection slopes and post-inflection slopes, as dependent variables), with Group (young participants vs. older participants) and Stimulus type (shapes vs. protrusions in Experiment 1; concavities vs. protrusions in Experiment 2) as fixed factors, and participant ID as random component. The LMM analysis was performed using the 1 me 4 and lmerTest packages (Bates et al., 2015; Kuznetsova et al., 2017) in the R environment for statistical computing. To evaluate the comparative evidence between the tested hypotheses, we also performed a Bayes factor analysis on the same data and variables using the R package BayesFactor (Rouder et al., 2012). In this latter analysis, all the relevant models (see Table 2) were contrasted against a null baseline model represented by the random component only. Finally, we additionally tested (conditioned on the main results obtained) more specific hypotheses according to a multiple comparison framework. All the analyses were performed using R 3.3.3 statistical environment (R Core Team, 2017).

\section{Results}

\section{Experiment 1}

Mean RTs of both groups as a function of numerosity for each stimulus condition are represented in Fig. 1B-C.

Overall model fitting The relation between the overall performance of the linear and bilinear models within each age group is depicted in Fig. 2A.

The graphical representation clearly shows that, for each participant in the young group (Fig. 2A, black dots), the overall goodness-of-fit of the estimated linear model was always smaller than the goodness-of-fit of the estimated bilinear model. In general, the distribution of adjusted $\mathrm{R}^{2}$ for the bilinear regression was significantly larger than that of the linear model on a Wilcoxon signed-rank test $(\mathrm{V}=0, p<0.0001)$. The same result was obtained by re-fitting the two models for each condition separately (protrusions vs. shapes) and for each individual in the young group. In both conditions the median adjusted $\mathrm{R}^{2}$ for the bilinear regression was significantly larger than the median adjusted $\mathrm{R}^{2}$ for the linear model on a Wilcoxon signed-rank test (protrusions : $\mathrm{V}=0, p<0.0001$; shapes: $\mathrm{V}=0, p<0.0001)$. Fully consistent results were obtained by considering the AIC and BIC indices.

Similar results were observed for the older group (Fig. 2A, gray dots). In fact, for the older group the performance of the bilinear model also dominated that of the linear regression as it was evident from the result of a Wilcoxon signed-rank test 
Table 2. Model coding for the Bayes factor analysis according to a linear mixed model representation (here Subject denotes the random component). $\mathrm{B}_{\mathrm{i} 0}$ indicates the Bayes factor representing the evidence of model [i] against the null model [0], with $\mathrm{i}=1,2,3$, and 4 . In general, $\mathrm{B}_{\mathrm{ij}}$ indicates the Bayes factor for the evidence of model [i] against model [j], with $\mathrm{j}=1,2,3$, and 4 . For substantive interpretation of Bayes factor results we used Jeffrey's ordinal recoding (1961)

\begin{tabular}{ll}
\hline Model code & Model structure \\
\hline [0] Null model & Y = Subject \\
[1] Main effect group & Y = Group + Subject \\
[2] Main effect stimulus & Y = Stimulus + Subject \\
[3] Main effects model & Y = Group + Stimulus + Subject \\
[4] Full model & Y = Group + Stimulus + Group $\times$ Stimulus + Subject \\
\hline
\end{tabular}

$(\mathrm{V}=0, p<0.0001)$. Analogous patterns were observed when considering submodels re-fitted on each condition separately (protrusions vs. shapes) and for each individual in the older
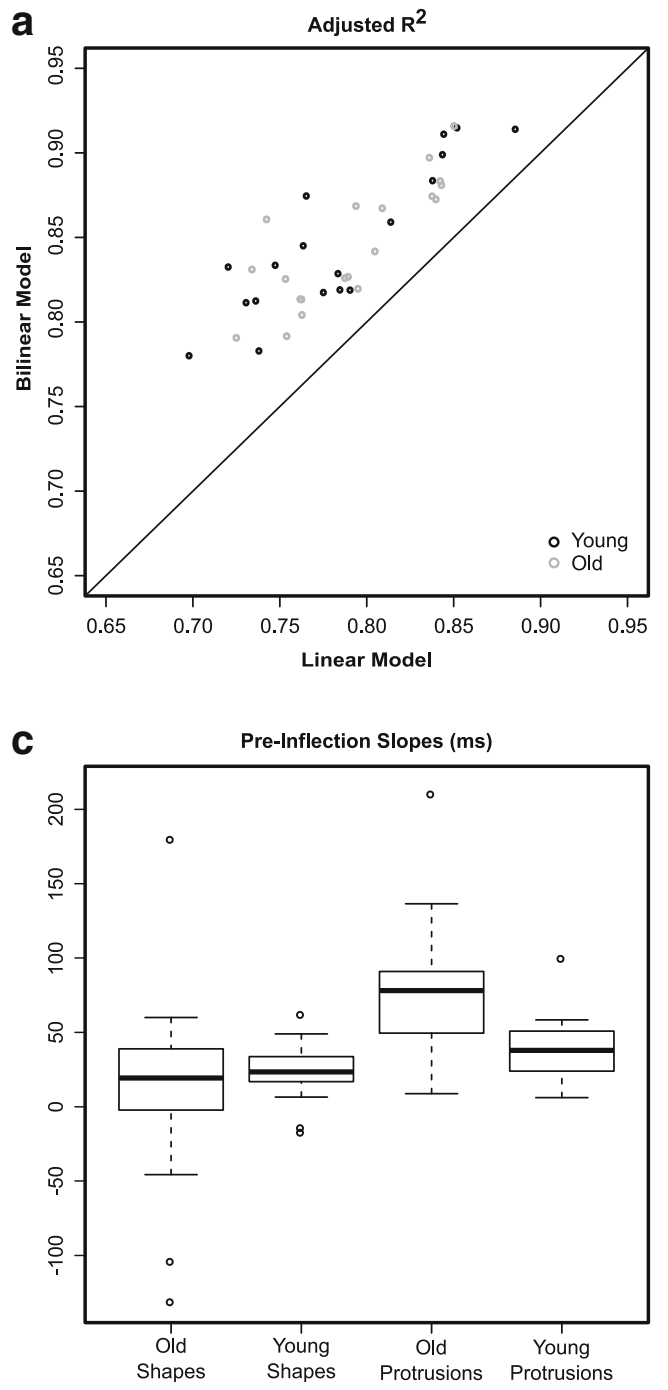

Fig. 2. Results of Experiment 1. (A) Relationship between the adjusted $\mathrm{R}^{2}$ computed on the estimated linear model and the adjusted $\mathrm{R}^{2}$ obtained using the estimated bilinear model for each single participant of both age groups; the diagonal segment indicates the hypothetical perfect 1:1 relationship (perfect identity) between the values depicted in the group. More specifically, for both conditions the median adjusted $\mathrm{R}^{2}$ of the bilinear regression was significantly larger than the median adjusted $\mathrm{R}^{2}$ for the linear model (protrusions:
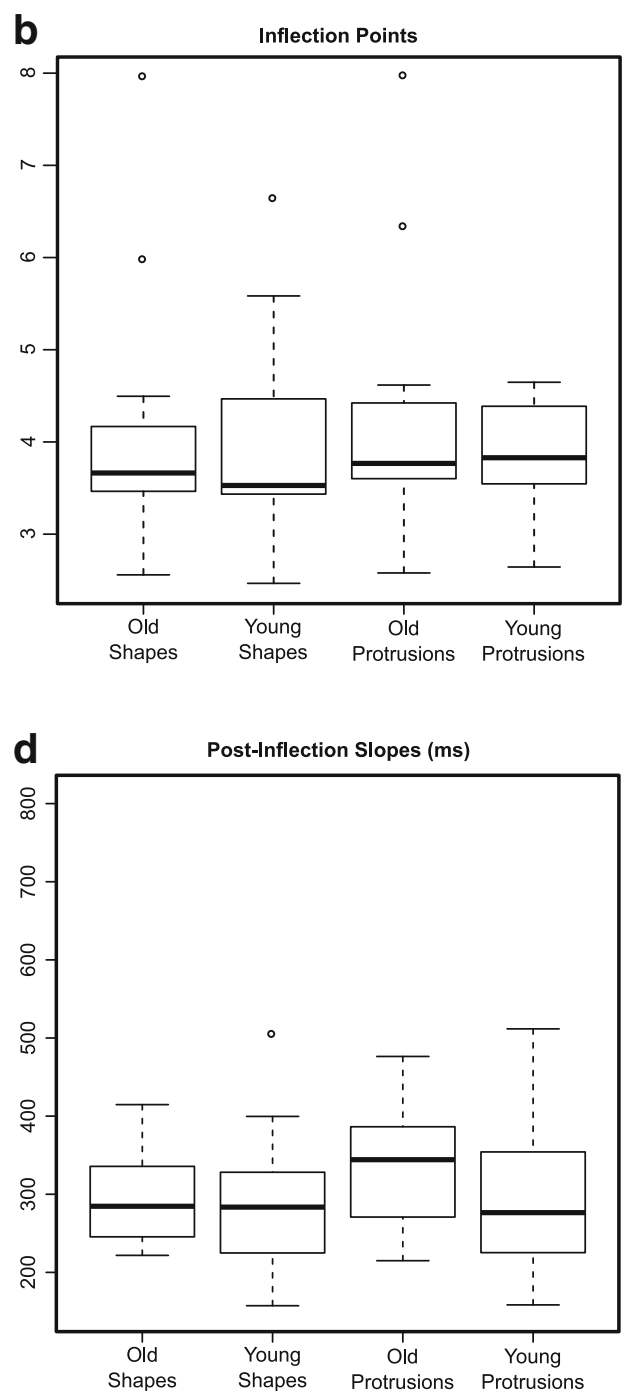

graphical representation for the two models. (B) Mean inflection points estimated for each group (old, young) and stimulus condition (protrusions, shapes) from the bilinear model. Mean (C) pre- and (D) post-inflection slopes for each group and stimulus condition estimated using the bilinear model 
$\mathrm{V}=0, p<0.0001$; shapes: $\mathrm{V}=0, p<0.0001)$. Similar results were observed with the AIC and BIC indices.

Because the bilinear model clearly outperformed the linear model, hereafter we will limit our analysis to the first representation only.

\section{Linear mixed model}

Inflection points The mean and median estimated inflection points for the two conditions are reported in Table 3. The results of the LMM applied on the inflection points provided by the fitted bilinear model showed that both the main effects and the interaction term (see also Fig. 2B) were not significant (all $p \mathrm{~s}>0.38$ in the analysis of variance derived from the output of the mixed linear model using the lmerTest package with Satterthwaite's degrees of freedom approximation). The same results were obtained with the Bayes factor analysis where all the four BFs considered (see Table 2) were associated with at least moderate evidence for the null model [0] (all $\mathrm{BFs}<0.3)$.

Pre-inflection slopes The results of the LMM applied on the pre-inflection slopes obtained by the fitted bilinear model showed a significant main effect for the Stimulus type factor $(F=18.8411, p<0.005)$, with protrusions showing larger preinflection slopes. The interaction term was also statistically significant $(F=7.4100, p<0.01$, see also Fig. 2 C). By contrast, the main effect of Group was not significant $(F=2.5475$, $p=0.1160$ ). Similar results were obtained with the Bayes factor analysis $\left(\mathrm{B}_{10}=0.5784, \mathrm{~B}_{20}=613.4481, \mathrm{~B}_{30}=\right.$ 449.717, $\mathrm{B}_{40}=3315.343$ ), in which the derived Bayes factor measures $\left(B_{43}=7.372, B_{42}=5.404\right)$ were consistent with moderate evidence for the interaction term, with protrusions being linked to a larger difference between the two groups of participants.

The multiple comparison analysis showed three significant results: (1) a significant difference between the two groups for the protrusion stimulus condition $(t(27.795)=-3.6233, p<$ 0.005 ), with elderly individuals having a larger pre-inflection slope; (2) within the young group, there was a statistically significant difference between shapes and protrusions $(t(17)$ $=2.3786, p=0.0294$ ); (3) a similar result was obtained for the older group $(t(19)=4.0632, p<0.001)$, with protrusions showing larger values for the pre-inflection slope. However, the Bayesian t-test analysis only supported the first $(\mathrm{BF}=$ $25.8935)$ and third $(\mathrm{BF}=52.3288)$ results. All the other comparisons were non-statistically significant (or with at least moderate evidence for the null model [0] on a Bayes factor analysis).

Post-inflection slopes The results of the LMM analysis showed a significant main effect for the Group factor $(F=$
4.030, $p=0.04952$, see also Fig. 2D), with elderly individuals being associated with larger values for the post-inflection slopes. However, the analysis performed using the Bayes factor only provided weak evidence for the grouping variable $\left(B_{10}=1.420\right)$. All the other results were somehow inconclusive $\left(B_{20}=0.3883, B_{30}=0.5564, B_{40}=0.2348\right)$, neither supporting the null or alternative models.

\section{Experiment 2}

Mean reaction times of both groups as a function of numerosity for each stimulus condition are shown in Fig. 3B-C.

Overall model fitting. The relation between the overall performance of the linear and bilinear models within each group is depicted in Fig. 4A. Data from one young participant were discarded from the analyses as the corresponding dataset resulted in unfeasible estimates for the bilinear model parameters. The results of the model fitting fully mirrored those reported for the first experiment. As for Experiment 1, because the bilinear model clearly outperformed the linear model, we will limit our analysis to the first representation only.

\section{Linear mixed model}

Inflection points The mean and median estimated inflection points for the two conditions are reported in Table 3. The results of the LMM applied on the inflection points provided by the fitted bilinear model showed that both the main effect of Group and the interaction term (see also Fig. 4B) were nonstatistically significant (all $p \mathrm{~s}>0.26$ ). By contrast, the main effect of stimulus was statistically significant $(F=4.509, p=$ 0.0385 ), with protrusions showing on average larger inflection points. However, this latter result was only mildly supported by the Bayes factor analysis $\left(B_{20}=2.262\right)$, which reported only weak evidence for the stimulus factor model [2] against the null model [0] (see Table 2).

Pre-inflection slopes The results of the LMM analysis applied on the pre-inflection slopes obtained by the fitted bilinear model showed a significant main effect for the factor Group $(F=28.8292, p<0.001)$ and for the interaction term $(F=$ 7.1527, $p=0.0099$, see also Fig. 4C). In particular, older individuals were associated with larger pre-inflection slopes. By contrast, the main effect of Stimulus was non-statistically significant $(F=1.4417, p=0.2353)$. Similar results were obtained with the Bayes factor analysis $\left(B_{10}=4974.438\right.$, $\left.\mathrm{B}_{20}=0.4190, \mathrm{~B}_{30}=2568.488, \mathrm{~B}_{40}=14732.43\right)$. However, the derived Bayes factor measures resulted in less clear results. 
a

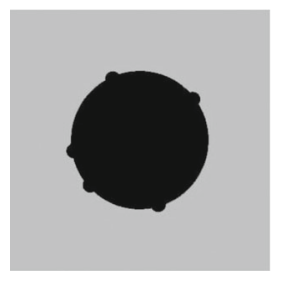

b

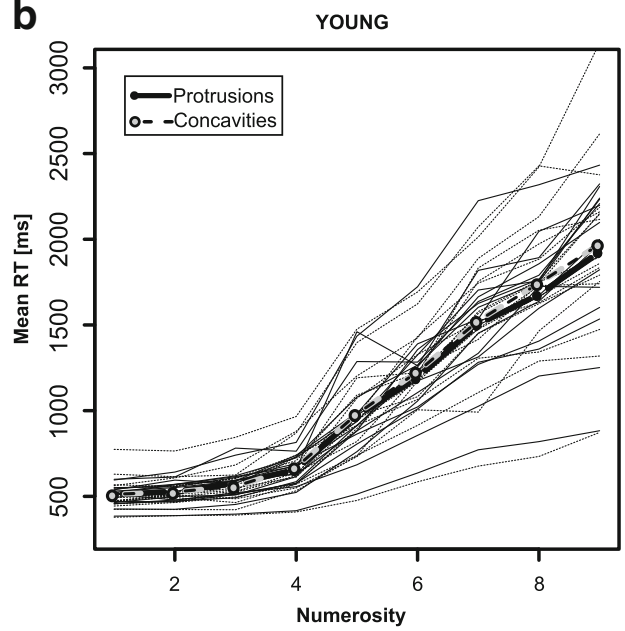

Fig. 3. (A) Example of stimuli for Experiment 2: protrusions (left) and concavities (right). Mean reaction times as a function of numerosity and stimulus condition for (B) young and (C) older participants. The thick

In particular, the evidence for the full model [4] against the main effects model [3] was associated with moderate evidence for the interaction term $\left(B_{43}=5.735\right)$. Nonetheless, the comparison of the full model [4] with the factor Group model [1] provided only weak evidence for [4] $\left(\mathrm{B}_{41}=2.96\right)$.

The multiple comparison analysis showed a significant difference between the two groups for the concavity stimulus condition $(t(30.246)=-5.1064, p<0.001$; see also Fig. $4 C)$, with older participants showing larger values for the preinflection slope. Within the same group, there was a statistically significant difference between concavities and protrusions $(t(19)=-2.7714, p=0.01216)$, with concavities being associated with larger pre-inflection slopes. The Bayesian version of the t-test returned very similar results. All the other comparisons were non-statistically significant in a t-test analysis (or with at least moderate evidence for the null hypothesis on a Bayes factor analysis).

Post-inflection slopes The results of the LMM showed significant main effects for both the Group factor $(F=34.090, p<$ $0.001)$ and the Stimulus factor $(F=4.9532, p=0.0304$; see also Fig. 4D for an interpretation of the results). In particular, the older group showed larger values for the post-inflection slopes, while concavities resulted in overall larger post-
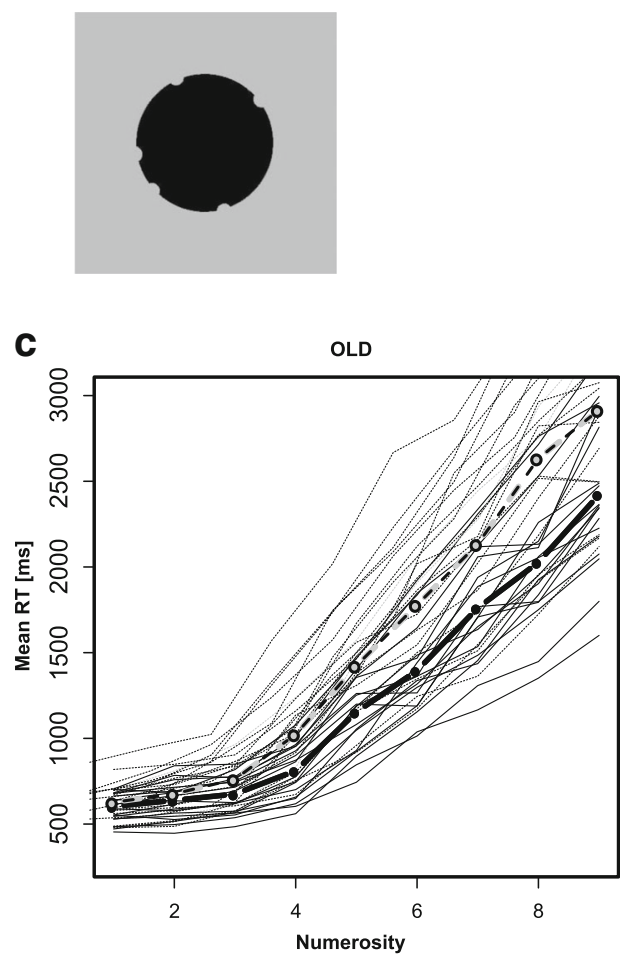

solid (protrusions) and segmented (concavities) lines represent the group average, while the thin solid and segmented lines represent each individual participant

inflection slopes than protrusions. By contrast, the interaction term was non-statistically significant $(F=2.7134, p=$ 0.1056 ). The Bayesian analysis was in general consistent with the former results $\left(\mathrm{B}_{10}=90564.86, \mathrm{~B}_{20}=1.296, \mathrm{~B}_{43}=0.939\right)$; however, for the Stimulus factor effect (model [2]) the Bayes factor resulted in a more conservative result denoting only weak evidence against the null model [0].

\section{Discussion}

Previous research has indicated that various perceptual (e.g., Schneider \& Pichora-Fuller, 2000) and cognitive (e.g., Park et al., 2002) abilities are subject to deterioration in aging. An interesting exception is represented by some enumeration tasks where efficient individuation is observed in both early and late adulthood (Pagano et al., 2016; Watson et al., 2002). However, while our knowledge about the factors allowing for efficient individuation in young adults has been enriched by a great deal of research, this level of detail is still lacking for aging. This study addressed whether spatial separability is a sufficient requirement for efficient individuation in late adulthood, as has been found for young adults. Various 

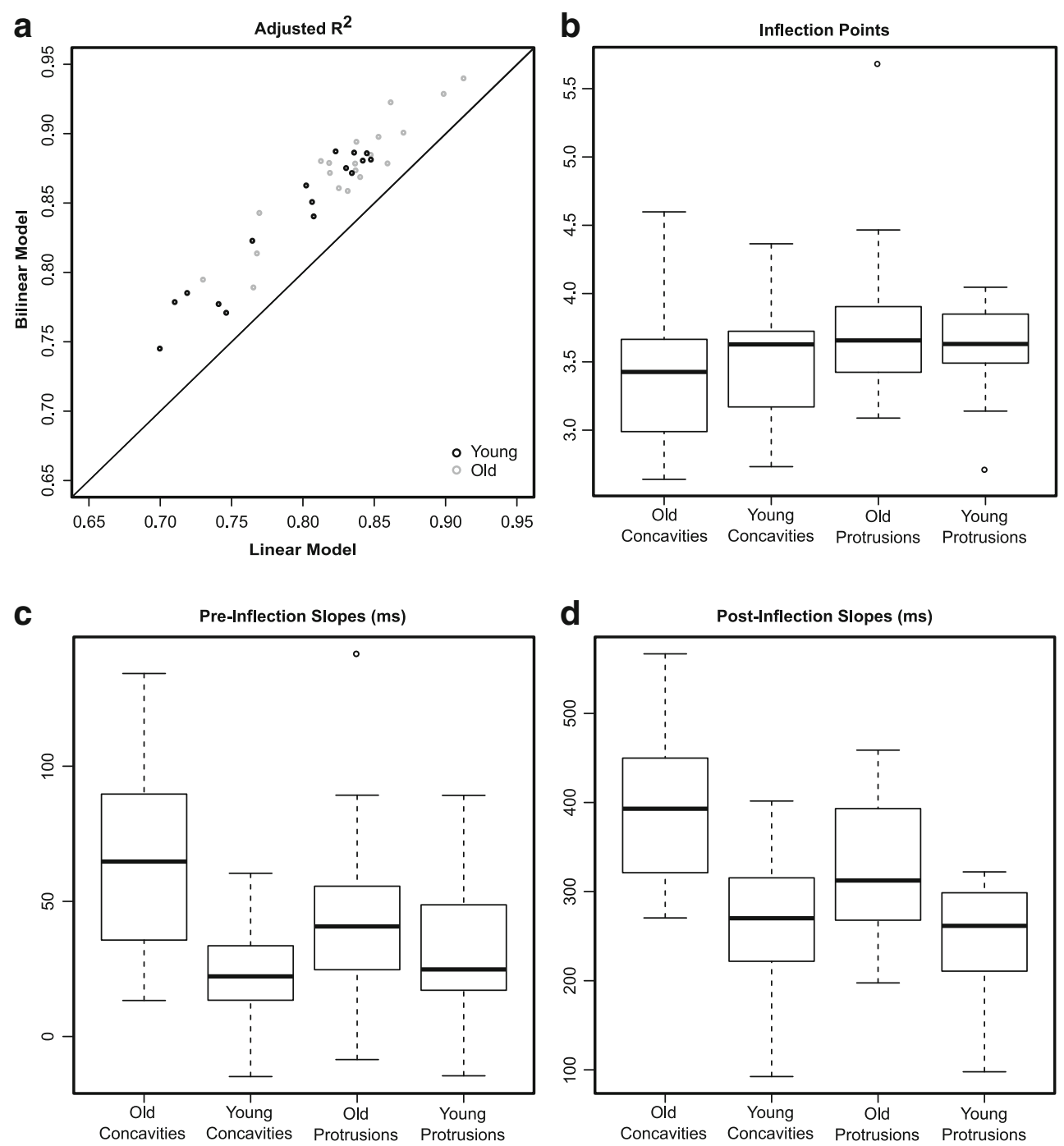

Fig. 4. Results of Experiment 2. (A) Relationship between the adjusted $\mathrm{R}^{2}$ computed on the estimated linear model and the adjusted $\mathrm{R}^{2}$ obtained using the estimated bilinear model for each single participant of both age groups; the diagonal segment indicates the hypothetical perfect 1:1 relationship (perfect identity) between the values depicted in the

Table 3. Mean and median estimated inflection points obtained by fitting the bilinear model on single participant data for each condition in the two experiments

\begin{tabular}{lllll}
\hline Experiment & Group & Condition & Mean (SD) & Median \\
\hline 1 & \multirow{2}{*}{ Young } & Protrusions & $3.86(0.59)$ & 3.83 \\
& & Shapes & $3.98(1.07)$ & 3.53 \\
& \multirow{2}{*}{ Old } & Protrusions & $4.15(1.17)$ & 3.77 \\
& \multirow{3}{*}{ Young } & Shapes & $3.91(1.23)$ & 3.66 \\
& \multirow{3}{*}{ Old } & Protrusions & $3.60(0.34)$ & 3.63 \\
& & Concavities & $3.49(0.46)$ & 3.63 \\
& & Protrusions & $3.79(0.59)$ & 3.66 \\
& & Concavities & $3.44(0.55)$ & 3.43 \\
\hline
\end{tabular}

graphical representation for the two models. (B) Mean inflection points estimated for each group and stimulus condition (concavities, protrusions) from the bilinear model. Mean (C) pre- and (D) postinflection slopes for each group and stimulus condition estimated using the bilinear model

aspects of the results were straightforward in highlighting the nature of object-part individuation in senescence.

In Experiment 1, the model-fitting procedure on the data of both groups indicated the typical elbow function (a proxy for subitizing) in response speed for both objects and object parts. This result indicates that subitizing is also present for object parts in older adults, just as in the case of disconnected objects (e.g., Trick et al., 1996). In addition, analysis of the inflection points revealed that for both groups the limit of efficient individuation of target elements was identified between three and four elements, both in the case of disconnected objects and in the case of object parts. Overall, and in line with Porter et al. (2016), these findings support the idea that spatial separability is a sufficient requirement for the emergence of subitizing across the lifespan. In addition, they suggest that the capacity 
limits of the individuation mechanism remain constant across the lifespan, with no substantial changes due to aging. The results also resonate with the evidence on early adulthood that attention can be deployed to object parts as efficiently as with unconnected objects (e.g., Marino \& Scholl, 2005; Vecera et al., 2000).

At variance with the analyses reported above, the results on the slope comparison revealed information related to stimulus-specific changes in enumeration speed due to aging. Indeed, older participants were penalized by object parts (compared to objects) for the small range of target numerosities (pre-inflection points). This suggests the presence of a small, stimulus-specific gradient in subitizing in the elderly. This age-specific finding may be related to the well-known phenomenon of age-related perceptual degradation in various dimensions, including the deterioration of contour detection (see Sekuler \& Sekuler, 2000). Indeed, in the task used in the present study the impairment shown by older individuals in perceiving contours efficiently may have resulted in difficulty in perceiving the various parts of a single object as distinct items, a requirement necessary for efficient enumeration. This, in turn, may have hindered the enumeration speed for object parts compared to disconnected objects, as seen in the present study for the subitizing range.

The finding that the effect of the stimulus nature was specific for the subitizing and not the counting range was relatively unexpected, as one may predict that age-related changes in perceptual abilities would encompass all item numerosities equally. However, previous research (Watson et al., 2005b, 2007) has indicated that a combination of factors, including increased noise or perceptual degradation, may specifically affect the speed for subitizing rather than counting numerosities. Thus, according to this research, the results of the present study indicate that some factors impact on only the first iteration of the individuation mechanism, while its subsequent reiteration (likely required for counting) may be less affected by the nature of the stimulus. Indeed, the operations required for counting (namely, reporting the exact number of elements when exceeding three to four units) are currently not fully understood, but the complexity of enumeration in this case with respect to small quantities (subitizing range) seems evident. For instance, counting may consist of a repeated reiteration of the individuation mechanism, with the resulting indexes added to the subsequent ones found at each reiteration (Trick \& Pylyshyn, 1993). As such, relative to subitizing, counting may require a stronger involvement of several cognitive functions (such as the active maintenance of the subtotals and/or running totals), which may be less susceptible to the effect of objecthood. Moreover, and in line with previous studies (e.g., Trick et al., 1996), results of both Experiment 1 and Experiment
2 found a general trend for an increase of enumeration slopes for the counting range in older participants, suggesting that slow processing speed represents a major component of the aging process (Salthouse, 1996, 2000). ${ }^{1}$ This overall slowing of enumeration speed for both objects and object parts (either concavities or protrusions) may in turn explain why the stimulus nature did not substantially influence older participants' performance in the counting range.

The purpose of Experiment 2 was to generalize the effects found for older adults by adopting a different set of stimuli (protrusions vs. concavities), similar to what has been shown in younger adults (Porter et al., 2016). As in Experiment 1, the results of the model-fitting procedure for both groups indicated the typical elbow function (a proxy for subitizing) in response speed for both objects and object parts. In addition, the results on the inflection points showed asymptote values that are compatible with the classic effects of subitizing in the literature (i.e., between three and four items, although the subitizing asymptote was slightly higher for protrusions than concavities in both groups), thus confirming that individuation functions over object parts just as efficiently as with distinct objects.

Comparison of the slopes for the subitizing range indicated that in older participants protrusions were easier to enumerate than concavities. This result is in line with some studies on curvature polarity in young individuals (Bertamini, 2001, 2003; Gibson, 1994) showing that convex shapes (i.e., protrusions) are perceived more efficiently than concave surfaces (i.e., concavities; although costs are found for different tasks, Hulleman et al., 1998; Humphreys \& Muller, 2000). Hence, in line with the results of Experiment 1, these findings suggest that only the first iteration of the individuation mechanism is substantially influenced by perceptual factors (such as curvature polarity) in aging. Future research will have to investigate this issue directly, as well as test whether and how curvature polarity per se undergoes age-related changes.

In conclusion, the current study indicates that individuation functions on both physically disconnected objects and separable object parts across the lifespan. The results on slope comparison (Experiment 1) indicate that some age-related changes in perceptual abilities may account for the difference in enumeration speed of disconnected versus connected items, suggesting a small advantage of objecthood for perception in aging. Additionally, perceptual factors such as curvature polarity seem to have an

\footnotetext{
${ }^{1}$ One explanation for this pattern could be related to a difference in the ability to apply attention efficiently in a particular pattern/strategy, where enumerating beyond four items would be achieved by starting at 12 o'clock and exploring the item systematically in a clockwise or anticlockwise manner - an ability that older adults may apply less efficiently.
} 
additional role in the enumeration speed of older persons (Experiment 2). However, as shown by the overall pattern of results of both experiments, spatial separation seems to be a sufficient requirement for efficient individuation that mostly resists degradation over time.

Acknowledgements The authors would like to thank Federica Platania, Valentina Brentari and Joseph Gabriele for support in data collection, and Katie Porter for providing the scripts.

Open Practice Statement None of the data or stimuli material of Experiments 1 and 2 are available online; however, they can be provided on request. The experiments were not preregistered.

\section{References}

Andersen, G. J., \& Ni, R. (2008). Aging and visual processing: Declines in spatial not temporal integration. Vision Research, 48, 109-118.

Atkinson, J., Campbell, E., \& Francis, M. (1976). The magic number $4 \pm$ 0: A new look at visual numerosity judgments. Perception, 5, 327334.

Bates, D., Maechler, M., Bolker, B., \& Walker, S. (2015). Fitting Linear Mixed-Effects Models Using lme4. Journal of Statistical Software, 67, 1-48.

Bertamini, M. (2001). The importance of being convex: An advantage for convexity when judging position. Perception, 30, 1295-1310.

Bertamini, M., \& Croucher, C. J. (2003). The shape of holes. Cognition, $87,33-54$.

Basak, C., \& Verhaeghen, P. (2003). Subitizing speed, subitizing range, counting speed, the Stroop effect, and aging: capacity differences and speed equivalence. Psychology and Aging, 18, 240-249.

Cavanagh, P. (2011). Visual cognition. Vision Research, 51, 1538-1551.

Del Viva, M. M., \& Agostini, R. (2007). Visual spatial integration in the elderly. Investigative Ophthalmology \& Visual Science, 48, 29402946.

Gibson, B. S. (1994). Visual attention and objects: one versus two or convex versus concave? Journal of Experimental Psychology: Human Perception and Performance, 20, 203-207.

Hulleman, J., Winkel, W., \& Boselie, F. (1998). Concavities as basic features in visual search: Evidence from search asymmetries. Perception \& Psychophysics, 62, 162- 174.

Humphreys, G., \& Muller, H. (2000). A search asymmetry reversed by figure-ground assignment. Psychological Science, 11, 196 -201.

Kahneman, D., Treisman, A., \& Gibbs, B. J. (1992). The reviewing of object files: Object-specific integration of information. Cognitive Psychology, 24, 175-219.

Kuznetsova, A., Brockhoff, P., \& Christensen, R. (2017). 1merTest Package: Tests in Linear Mixed Effects Models. Journal of Statistical Software, 82, 1-26.

Mandler, G., \& Shebo, B. J. (1982). Subitizing: an analysis of its component processes. Journal of Experimental Psychology: General, $111,1-22$

Marino, A. C., \& Scholl, B. J. (2005). The role of closure in defining the "objects" of object-based attention. Perception \& Psychophysics, 67, 1140-1149.

Mazza, V., \& Caramazza, A. (2011). Temporal brain dynamics of multiple object processing: the flexibility of individuation. PLoS One, 6, e 7453.

Maylor, E. A., Sheehan, B., Watson, D. G., \& Henderson E. L. (2008). Enumeration in Alzheimer's disease and other late life psychiatric syndromes. Neuropsychologia, 46, 2696-2708.
Maylor, E. A., Watson, D. G., \& Muller, Z. (2005). Effects of Alzheimer's disease on visual enumeration. The Journals of Gerontology. Series B, Psychological Sciences and Social Sciences, 60, 129-135.

Monge, Z. A., \& Madden, D. J. (2016). Linking cognitive and visual perceptual decline in healthy aging: The information degradation hypothesis. Neuroscience \& Biobehavioral Reviews, 69, 166-173.

Muggeo, V. M. R. (2008). segmented: An R package to fit regression models with broken-line relationships. $R$ News, 8, 20-25.

Pagano, S., Fait, E., Brignani, D., \& Mazza, V. (2016). Object individuation and compensation in healthy aging. Neurobiology of Aging, 40, 145-154.

Park, D.C., Lautenschlager, G., Hedden, T., Davidson, N.S., Smith, A.D., \& Smith, P.K. (2002). Models of visuospatial and verbal memory across the adult life span. Psychology and Aging, 17, 299-320.

Poncet, M., Caramazza, A., \& Mazza, V. (2016). Individuation of objects and object parts rely on the same neuronal mechanism. Scientific Reports, 6, 38434.

Porter, K. B., Mazza, V., Garofalo, A., \& Caramazza, A. (2016). Visual object individuation occurs over object wholes, parts, and even holes. Attention, Perception, \& Psychophysics, 78, 1145-62.

Pylyshyn, Z. W. (2001). Visual indexes, preconceptual objects, and situated vision. Cognition, 80, 127-158.

R Core Team (2017). R: A language and environment for statistical computing. R Foundation for Statistical Computing, Vienna, Austria. URL https://www.R-project.org/.

Rabbitt, P. (2016). Speed of Visual Search in Old Age: 1950 to 2016. The Journals of Gerontology. Series B, Psychological Sciences and Social Sciences, 72, 51-60.

Roudaia, E., Bennett, P. J., \& Sekuler, A. B. (2008). The effect of aging on contour integration. Vision Research, 48, 2767-2774.

Roudaia, E., Farber, L. E., Bennett, P. J., \& Sekuler, A. B. (2011). The effects of aging on contour discrimination in clutter. Vision Research, 51, 1022-1032.

Rouder, J. N., Morey, R. D., Speckman, P. L., \& Province, J. M., (2012) Default bayes factors for ANOVA designs. Journal of Mathematical Psychology, 56, 356-374.

Schneider, B. A., \& Pichora-Fuller M. K. (2000). Implications of perceptual deterioration for cognitive aging research. In F. I. M. Craik \& T. A. Salthouse (Eds.), The handbook of aging and cognition (pp. 155219). Mahwah, NJ, US: Lawrence Erlbaum Associates Publishers.

Salthouse, T. A. (1996). The processing-speed theory of adult age differences in cognition. Psychological Review, 103, 403-428.

Salthouse, T. A. (2000). Aging and measures of processing speed. Biological Psychology, 54, 35-54.

Salthouse, T. A. (2004). What and when of cognitive aging. Current Directions in Psychological Science, 13, 140-144.

Sekuler, R., \& Sekuler, A. (2000). Visual Perception and Cognition. In oxford Textbook of Geriatric Medicine (pp. 874-880), J.G. Evans et al. (Eds). Oxford University Press.

Trick, L. M., \& Pylyshyn, Z. W. (1993). What enumeration studies can show us about spatial attention: evidence for limited capacity preattentive processing. Journal of Experimental Psychology: Human Perception and Performance, 19, 331-351.

Trick, L. M., \& Pylyshyn, Z. W. (1994). Why are small and large numbers enumerated differently? A limited-capacity preattentive stage in vision. Psychological Review, 101, 80-102.

Trick, L. M., Enns, J. T., \& Brodeur, D. A. (1996). Life span changes in visual enumeration: The number discrimination task. Developmental Psychology, 32, 925-932.

Vecera, S. P., Behrmann, M., \& McGoldrick, J. (2000). Selective attention to the parts of an object. Psychonomic Bulletin \& Review, 7, 301 308.

Vetter, P., Butterworth, B., \& Bahrami, B. (2008). Modulating attentional load affects numerosity estimation: evidence against a pre-attentive subitizing mechanism. PLoS One, 3, e3269. 
Watson, D. G., \& Humphreys, G. W. (1999). The magic number four and temporo-parietal damage: neurological impairments in counting targets amongst distractors. Cognitive Neuropsychology, 16, 609-629.

Watson, D. G., Maylor, E. A., Allen, G. E. J., \& Bruce, L. A. M. (2007). Early visual tagging: Effects of target-distractor similarity and old age on search, subitization, and counting. Journal of Experimental Psychology: Human Perception and Performance, 33, 549-569.

Watson, D. G., Maylor, E. A., \& Bruce, L. A. M. (2005a). The efficiency of feature-based subitization and counting. Journal of Experimental Psychology: Human Perception and Performance, 31, 1449-1462.

Watson, D. G., Maylor, E. A., \& Bruce, L. A. M. (2005b). Search, enumeration, and aging: eye movement requirements cause age- equivalent performance in enumeration but not in search tasks. Psychology and Aging, 20, 226-240.

Watson, D.G., Maylor, E.A., \& Manson, N.J. (2002). Aging and enumeration: a selective deficit for the subitization of targets among distractors. Psychology and Aging, 17, 496-504.

Wurm, M. F., Porter, K. B., \& Caramazza, A. (2019). Individuation of parts of a single object and multiple distinct objects relies on a common neural mechanism in inferior intraparietal sulcus. Cortex, $121,1-15$.

Publisher's note Springer Nature remains neutral with regard to jurisdictional claims in published maps and institutional affiliations. 